\title{
A Kind of Differentiated Business Sense RWA Based on Wavelength Layered Graph
}

\author{
Weihong Cai ${ }^{1, a}$, Hongjuan Liu ${ }^{2, \mathrm{~b}}$ and Li Du ${ }^{3, \mathrm{c}}$ \\ ${ }^{1}$ Key Laboratory of Medical Image Computing of Ministry of Education, Northeastern University, \\ Shenyang, 110819, China \\ ${ }^{2}$ Key Laboratory of Medical Image Computing of Ministry of Education, Northeastern University, \\ Shenyang, 110819, China \\ ${ }^{3}$ School of Information Science and Engineering, Northeastern University, Shenyang, 110819, \\ China \\ ahongjuanliu03@163.com, bhongjuanliu03@163.com, 'duli26@126.com
}

Keywords: IONs, wavelength layered graph, LWG, LWGB, wavelength buffering.

Abstract. The network congestion problem and differentiated business are important factors to impact the performance of network. This paper studies the RWA of differentiated business based on the wavelength layered graph model in the IONs and puts forward some designs. Firstly, combined dynamic weight formula with wavelength layered graph. Secondly, design wavelength layered grouping strategy. Thirdly, wavelength buffering is introduced not only to guarantee high-priority business blocking performance but also to reduce the blocking rate of low-priority business. Simulation result shows that LWGB algorithm achieved an outstanding performance of the network.

\section{Introduction}

Internet has brought the revolutionary changes for the world, the emerging voice, video and other multimedia business on the network have put forward higher requirements. With the optical network from the carrier network to the business network evolution, RWA related technology to dynamically adapt to the characteristics of network business perception. RWA algorithms have been proposed are mostly based on static network business, and most researches on RWA algorithms focus on optimizing the number of wavelengths used and reducing network congestion rate, but how to differentiated business of network and reduce the high-priority business blocking rate is relatively few. Therefore, in the process of differentiated business needs routing and wavelength assignment, not only to consider the use of wavelength and the blocking rate of network, but also taken into account the dynamic nature of the business and differentiated business needs and adjust accordingly RWA algorithms.

\section{Problem Statement}

Link cost function. Assume the physical topology is $G=(V, E, W)$, where $V$ is the set of nodes, $E$ is the set of unidirectional fiber links, and $\mathrm{W}$ is the set of wavelengths supported in a fiber link. $|\mathrm{V}|$, $|\mathrm{E}|$ and $|\mathrm{W}|$ indicate the number of nodes, the number of links and the number of wavelengths respectively.

Constraints. Wavelength continuity constraint: in a network without wavelength conversion facility, all the links in a path traverse must be use the same wavelength.

Capacity Constraint: The total bandwidth of all the connections carries over a lightpath must less than the bandwidth of a lightpath.

Transceiver Constraint: To establish a lightpath, the source node must have an available transmitter and the destination node must have an available receiver. 


\section{LWGB Algorithm Design}

This paper introduced wavelength layered graph for business routing and wavelength assignment to avoid the wavelength continuity constraint. In order to differentiated business of network and guarantee the blocking performance of high-priority business. This paper proposes the wavelength layered grouping strategy and designs the LWGB as the differentiated business routing algorithm.

Strategies of dynamic weights based on wavelength layered graph. In order to the network have a balanced load distribution, reduce the network congestion rate and improve the performance of network, this paper introduces strategies of dynamic weights based on wavelength layered graph (LW). Dynamic weight is calculated as follows.

Specifically, this paper uses the product of the number of LSP $\mathrm{N}_{\mathrm{LSP}}$ and the physical length of link $\mathrm{D}$ to calculate the criticality weight $\mathrm{W}_{\mathrm{C}}$, defined as Eq. 1:

$$
W_{C}=N_{L S P} \times D
$$

In the process of calculating the link load weight, it uses the link load rate $\mathrm{P}_{\text {load}}$, defined as Eq. 2:

$$
P_{\text {load }}=\frac{B_{\text {used }}}{B_{\text {total }}}
$$

Where $\mathrm{B}_{\text {total }}$ denotes the total bandwidth of a link. $\mathrm{B}_{\text {used }}$ denotes the bandwidth which has been used. Therefore, link load weight $\mathrm{W}_{\mathrm{L}}$ can be calculated as Eq. 3:

$$
W_{L}=\frac{P_{\text {load }}^{\alpha}}{1-P_{\text {load }}^{\alpha}} \times N_{L S P} \times D
$$

Therefore, the dynamic weight $\mathrm{W}$ based on wavelength layered is defined as Eq. 4 :

$$
W=\left(\frac{P_{\text {load }}^{\alpha}}{1-P_{\text {load }}^{\alpha}}+1\right) \times N_{L S P} \times D
$$

Where $\alpha>1$, and its specific value determines the influence of the link load weight for the weight of a link.

Wavelength layer grouping strategy design on LW. In order to differentiated business of network, guarantee business of each grade have available resources and reduce the high-priority business blocking rate, this paper proposed wavelength layer grouping strategy. The strategy takes the wavelength layered into three parts according to 1:1:1 proportion. Assuming that the division of three groups of wavelengths correspond to the network layer resources of high, medium and low grade business, high-priority business not only can conduct business in their allocation of resources in the corresponding packet, also can rent unused resources of medium-priority and low-priority resources grouping, medium-priority business not only can conduct business in their allocation of resources in the corresponding packet, also can rent unused resources low-priority's resources grouping. Wavelength layer grouping strategy not only can distinguish the network business, also can protect high-priority business blocking performance. The strategy based on LW called LWG.

Wavelength buffering scheme on LWG. In order to improve network performance, guarantee blocking performance of high-priority business and decrease the blocking probability of low-priority business, this paper introduced wavelength buffering scheme based on LWG called LWGB.

The main idea of wavelength buffering scheme is to set a wavelength buffer in the network, and put some wavelengths in the zone, when the whole network traffic load is low, the paper just start routing and wavelength assignment in the non-wavelength buffer, and in the peak phase of the traffic load "release" the resources in wavelength buffer to improve the network resource utilization and reduce the network blocking probability.

Setting of wavelength buffering: 
The size of the wavelength buffer has a direct impact on the network optimization. If the size is too large or too small, it cannot improve performance of network.

Step 1: Assume all the network traffics are high-priority.

Step 2: For the given network traffic load, wavelength buffer increases from zero, and increases its minimum size every time.

Step 3: When the wavelength buffer has increased to a fixed value, observe the change of blocking probability of the whole network until the fluctuation range less than $1 \%$ of its value, record the blocking probability. And continue to increase wavelength buffer. Repeat the operation of Step 3 until the emergence of the recorded minimum value of blocking probability.

Step 4: Find the smallest blocking probability in the recorded values, and the corresponding buffer capacity is the optimal wavelength buffer.

Step 5: Increase network traffic loads, repeat Step 2 to Step 4 until blocking probability indicator is over the upper limit of its value. In general, this limit can be set $20 \%$. Finally, the paper get the optimal wavelength buffer trends with traffic load.

Starting of wavelength buffering:

In order to make better use of the network resource in wavelength buffer, only high-priority business and low-priority business meeting certain conditions can start wavelength buffer. To low-priority traffic, it should meet two conditions.

Instantaneous traffic load I is greater than the setting threshold. As shown in Eq. 5:

\section{$I=$ InsTrafNum $/ N$}

Where, InsTrafNum is the instantaneous traffic number of the network.

The rate of resources utilization of wavelength buffer is less than the setting threshold. As shown in Eq. 6.

$$
I=|E|^{-1} \sum_{i=1}^{|E|} \sum_{j=1}^{k} e_{i j} / k
$$

Where, $e_{i j}$ is the state of the link $i$ in wavelength buffer in its $j$ times. If the link is busy, $e_{i j}=1$, otherwise $e_{i j}=0$. $|E|$ denotes the amount of wavelength in wavelength buffer, k denotes the times of network resource utilization statistics.

LWGB algorithm. The basic procedure of LWGB algorithm:

Step 1: Generating wavelength layered graph.

Step 2: Calculating wavelength layered buffer, and add corresponding amount of wavelength layered in wavelength buffer. Dividing wavelength layered into three parts according to 1:1:1 proportion of non-wavelength buffer.

Step 3: Whether the level of new business is high-priority, for high-priority, go to Step 4, else, go to Step 5.

Step 4: Dynamic to find the wavelength grouping G1of high-priority in non-wavelength buffer, and use the dynamic Dijkstra algorithm to find the optical route for the business, if there is an available path, go to Step 11, else, go to Step 6.

Step 5: Whether the level of new business is medium-priority, for medium-priority, go to Step 6, else, go to Step 7.

Step 6: Dynamic find the wavelength grouping G2 of medium-priority in non-wavelength buffer, and use the dynamic Dijkstra algorithm to find the optical route for the business, if there is an available path, go to Step 11, else, go to Step 7.

Step 7: Dynamic find the wavelength grouping G3 of low-priority in non-wavelength buffer, and use the dynamic Dijkstra algorithm to find the optical route for the business, if there is an available path, go to Step 11, else, go to Step 8.

Step 8: According to pre-setting level business priorities, for low-priority business, go to Step 9, for high-priority and medium-priority business, go to Step 10.

Step 9: If the instantaneous business load of the network is greater than the given threshold, and the network resource utilization of wavelength buffer is less than the given threshold, go to Step 10, 
else, go to Step 4.

Step 10: Start wavelength buffering, Routing and wavelength assignment for the high-priority and medium-priority business in Step 8 or low-priority business meets the conditions in Step 9 in wavelength buffer with Dijkstra and First-Fit algorithm. If existing the shortest route and wavelength assignment success, calculate the network resource utilization of wavelength buffer, go to Step 11, if not, output congestion information and go to Step 12.

Step 11: Allocation network resources for business, and update wavelength layered graph, go to step 12.

Step 12: If all the business requests finish routing, go to step 13, else, go back Step 3.

Step 13: Algorithm ends.

Simulation. In the simulation, the paper use the NSFNet network topology which composed of 14 nodes and 21 links, is shown in Fig. 1.

In the simulation, the paper test the blocking probability, end to end delay, network resource utilization, blocking probability of each-grade business. In order to test the performance of dynamic weight formula, wavelength layered grouping and wavelength buffering scheme in the LWGB algorithm, the paper design some schemes which are D+FF, LW, LWG and LWGB algorithm.

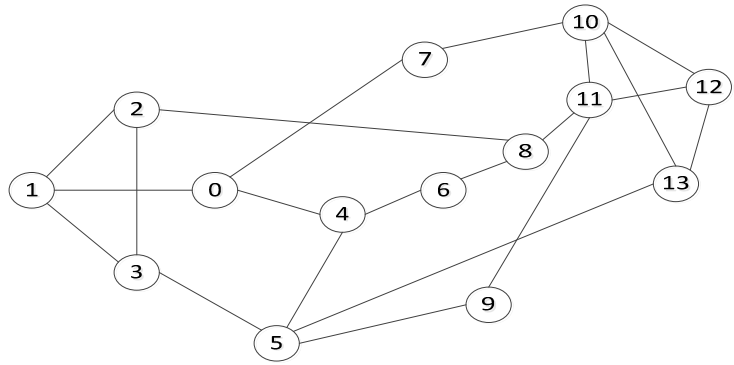

Fig. 1. NSFNet network

Fig. 2 compares the blocking probability of D+FF, LW, LWG and LWGB. It can conclude that the blocking probability of LWGB is smaller than other algorithm. This is because the LWGB algorithm can let more business transit in the network.

Fig. 3 shows that with the increasing of network traffic load, end-to-end delay becomes more and more large. It can conclude that the LWGB algorithm delay lowest. There are two reasons for this phenomenon: 1) LWGB algorithm make more business transit in the network; 2) the existence of wavelength buffering can decrease the blocking probability of the high-priority business and the low-priority business which meet the conditions.

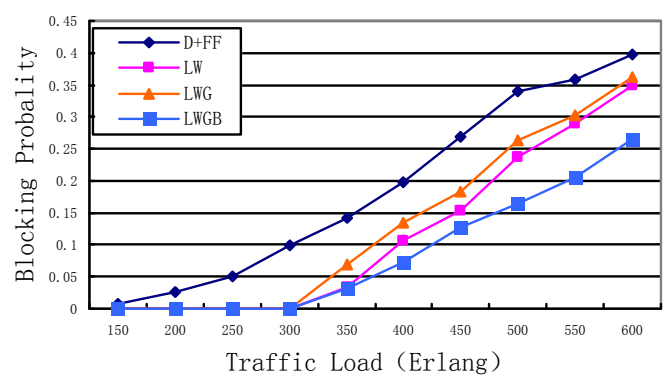

Fig. 2. Comparison of blocking probability

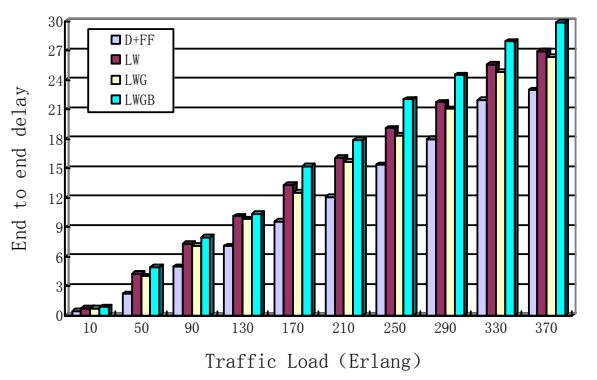

Fig. 3. Comparison of end-to end delay

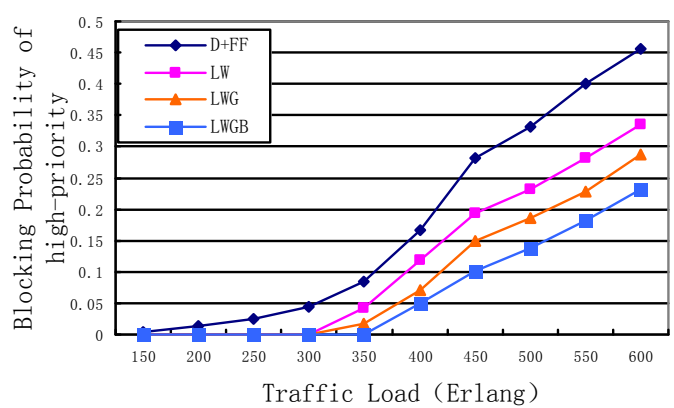

Fig. 4. Comparison of high-priority business Congestion probability 


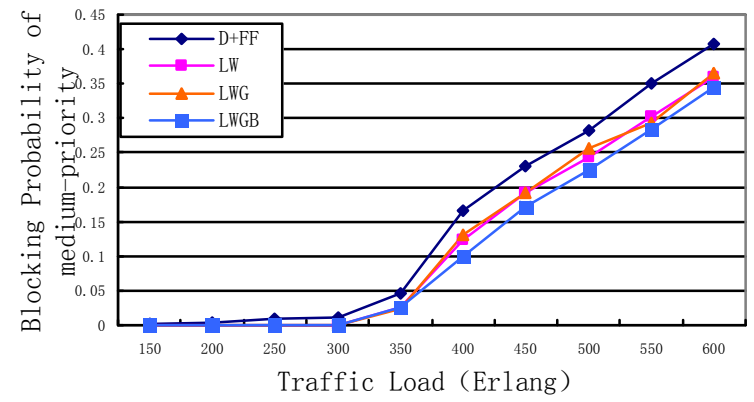

Fig. 5. Comparison of medium-priority business Congestion probability

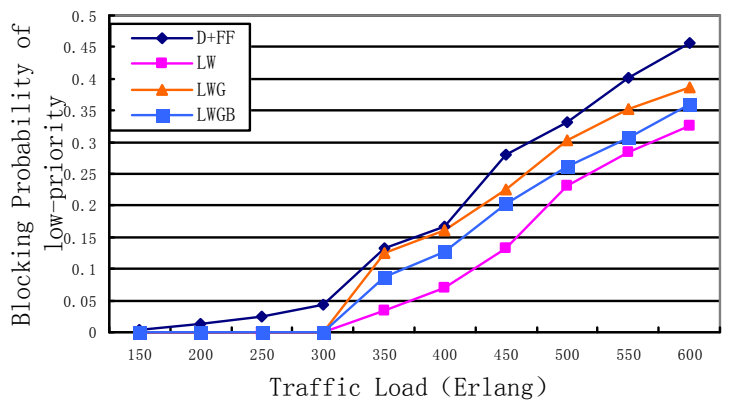

Fig. 6. Comparison of low-priority business Congestion probability

Fig. 4, Fig. 5 and Fig. 6 shows that LWGB algorithm decrease the blocking probability of high priority business and medium priority business, but the blocking probability of low priority business is increase. This is because LWGB algorithm can let high priority and medium priority hire unused resources of low priority, so LWGB algorithm at the expense of some blocking performance of low priority business to protect high-priority blocking performance. LWGB algorithm not only can differentiated business also can guaranteeing blocking performance of high-priority.

\section{Conclusions}

This paper analyses differentiated business routing algorithm in IONs. In order to improve the performance of network and minimize the high-priority blocking probability, the paper proposed two algorithms: LWG and LWGB. LWG algorithm can reduce the blocking rate of high priority business 6\%; LWGB algorithm can reduce the blocking rate of high priority and improve the network resource utilization effectively, the network blocking probability reduces $10 \%$. Simulation results show that wavelength layered graph, wavelength layered grouping and wavelength buffering scheme improve the performance of the LWGB algorithm in reducing network blocking probability and blocking probability of high-priority business aspect.

\section{Acknowledgements}

This research was financially supported by the Key Laboratory of Medical Image Computing of Mi nistry of Education, Northeastern University.

\section{References}

[1] Barpanda R S, Turuk, A K, Sahoo B, ect. Genetic Algorithm techniques to solve Routing and Wavelength Assignment problem in Wavelength Division Multiplexing all-optical networks [A]. 2011 Third International Conference on Comm. Systems and Networks (COMSNETS)[C]. 2011:1-8.

[2] Gao D, Zhang H. Simulation study on the interactivity of failure detection and signaling congestion control in intelligent optical networks[J]. Photonic Network Communications, 2007, 
14(2): 149-157.

[3] Hemmati H, Biswas A, Djordjevic I B. Deep-space optical communications: Future perspectives and applications[J]. Proceedings of the IEEE, 2011, 99(11): 2020-2039.

[4] Huang H, Zhang J, Xia L, et al. Dynamic weight protection ring apply to multi-failures in WDM optical networks[A], 2011 International Conference on Information Photonics and Optical Communications[C],Jurong West,2011,1-4.

[5] Loi C H Liao W. Multiclass wavelength reservation in optical burst switched WDM networks. In: Proc. of IEEE International Conference on Communications, Circuits and Systems and West Sino Expositions, 2008: 845-849.

[6] Parra O J S, Rubio G L, Castellanos L. Mechanisms to Ensure QoS over ASON[A]. 2011 International Joint Conference on Service Sciences(IJCSS)[C],Taipei,2011,203-207.

[7] Rahbar A G P. Dynamic impairment-aware RWA in multifiber wavelength-routed all-optical networks supporting class-based traffic [J]. Optical Communications and Networking, 2010, 2(11): 915-927. 